\title{
Advantages of Anisotropic Wheels in Skid Steer Turns
}

\author{
Daniel Flippo $^{1 *}$ and David P. Miller ${ }^{2}$ \\ ${ }^{1}$ Kansas State University, Manhattan KS \\ ${ }^{2}$ University of Oklahoma, Norman $\mathrm{OK}$
}

Received: April 23, 2015; Accepted: February 12, 2016; Published: February 18, 2016

*Corresponding author: Daniel Flippo, Kansas State University, Manhattan KS, 66502; E-mail: dkflippo@ksu.edu

\begin{abstract}
Wheels are the conventional mode of locomotion, especially for planetary exploration robots. These missions require long distances on limited power over discontinuous terrain. These requirements make the wheel a major component contributing to performance. Any enhancement that can be made to a wheel will enhance the total vehicle's performance in that area. One area of interest is skid steer turning, which is a simple practical approach to vehicle design. A disadvantage of skid steering is the low efficiency (angle turned per unit of energy) in turning due to the necessary sliding of the wheel in the lateral direction. This paper specifically studies skid steer turning and a way to increase efficiency by decreasing the lateral frictional force in relation to longitudinal frictional force. The concept is then tested using an anisotropic wheel that illustrates this behavior.
\end{abstract}

\section{Introduction}

Skid steering is a design methodology in vehicles to maneuver by simply incurring a velocity difference between its two lateral sides. Modern wheel skid steer loaders illustrate the concept in the commercial world; while caterpillar tracked combat vehicles with the tracked skid steer variation. Skid steering does not need a steering mechanism so its simplicity is attractive as well as its ability to spin from its center axis gives skid steering high mobility [7]. Due to skidding some drawbacks include high wear and tear of the components, the energy loss from skidding, and cause damage to the soil. If turning efficiency can be improved then skid steering would be more useful in maneuver intensive mission applications [3].

\section{Theory of Turn Rate vs Wheel Force}

- $\Omega=$ Vehicle skid steer spin rate in a skid steer turn.

- $\quad \mathrm{M}_{\text {friction }}=$ The moment incurred on a vehicle from the soil frictional forces.

- $\mathrm{M}_{\text {motor }}=$ The moment incurred on a vehicle from the motor power.

- $\mathrm{M}_{0}=$ The total moment of the vehicle in the XY plane.

- $\mathrm{R}_{\mathrm{Y}}=$ The distance from the center of the vehicle to the axle of the wheel longitudinally.

- $\mathrm{R}_{\mathrm{x}}=$ The distance from the center of the vehicle to the middle of the wheel laterally.

- $\mathrm{R}=$ The distance from the center of the vehicle to the center of the wheel.

- $\omega=$ The wheel spin rate in radians/second.

- $\mathrm{F}_{\mathrm{Y}}=$ The total net force in the longitudinal direction on one wheel.

- $\mathrm{F}_{\mathrm{X}}=$ The total net force in the lateral direction on one wheel.

- $\mathrm{F}_{\mathrm{y}}=$ The traction force in the longitudinal direction on one wheel.

- $\mathrm{F}_{\mathrm{x}}=$ The traction force in the lateral direction on one wheel.

- $\mathrm{f}_{\mathrm{y}}=$ The frictional force in the longitudinal direction on one wheel.

- $\mathrm{f}_{\mathrm{x}}=$ The frictional force in the lateral direction on one wheel.

- $Q=$ The angle that the front wheel is placed off of the center of the vehicle.

Skid steering is an unintuitive method from lateral sliding that must take place for a skid steer vehicle to turn steering due to the multiple forces. When a skid steered vehicle initiates a turn, its rotation in the $(\mathrm{X}-\mathrm{Y})$ plane will accelerate up due to a certain spin rate $\Omega$ Figure 1 . In this point it will stabilize and the total moment about its center $\left(\mathrm{M}_{\mathrm{o}}\right)$ will equal zero and the resistive moment $\mathrm{M}_{\text {friction }}$ will cancel out the moment induced by the motors $\left(\mathrm{M}_{\text {motor }}\right)$. $\mathrm{M}_{\text {friction }}$ consists of the frictional forces due to sliding, offset by $\mathrm{R}_{\mathrm{Y}}$ on each wheel Equation 1.

$$
\begin{aligned}
& \mathrm{M}_{\text {friction }}=\sum \mathrm{F}_{\mathrm{X}} \mathrm{R}_{\mathrm{Y}}=\sum \mathrm{SF}_{\mathrm{x}} \mathrm{R} \sin \theta . \\
& \mathrm{M} \text { motor }-\mathrm{M} \text { friction }=\mathrm{Mo}=0 . \\
& \sum\left(\mathrm{F}_{\mathrm{y}} \mathrm{R} \cos (\theta)\right)-\sum\left(\mathrm{F}_{\mathrm{x}} \mathrm{R} \sin (\theta)\right)=0 . \\
& \mathrm{F}_{\mathrm{y}}=\mathrm{F}_{\mathrm{x}} \tan (\theta) .
\end{aligned}
$$




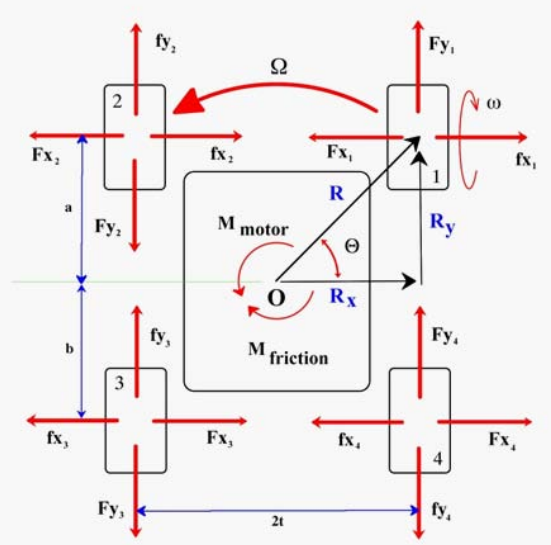

Figure 1: Force Body Diagram of a generalized skid steer.

Flippo, et.al. [5] use Equation 2 through 4 derive a relationship between $\mathrm{F}_{\mathrm{x}}$ and $\mathrm{F}_{\mathrm{y}}$ at the turning equilibrium point and is dependent upon the vehicle geometry $(\theta)$. If the vehicle were slender Figure 2A then $\theta$ would be larger than $\frac{\pi}{2}$ an Equation 4 would dictate that $\mathrm{F}_{\mathrm{x}}$ would be much smaller than $\mathrm{F}_{\mathrm{y}}$. If $\theta=$ $\frac{\pi}{2}$ then $\mathrm{F}_{\mathrm{y}}$ would approach infinity, making the force exerted by the wheel independent of the vehicle's spin rate $\Omega$ which would always be zero. If, on the other hand, $\theta$ were equal to zero, as in Figure $2 \mathrm{~B}$, then $\mathrm{F}_{\mathrm{y}}$ (which is really the net force of applied force and friction) would be zero. This would equal the Ackerman steering method which means that the wheels have no lateral slip. If there is also no longitudinal slip then the turning rate can be calculated by Equation. 5

$$
\Omega=\frac{\omega r}{R}, F y=0 .
$$

The wheel angular spin velocity $\omega$ is in radians per second, and $\mathrm{R}$ is the distance from the center of the footprint of the wheel to the center of the vehicle's rotation, and $r$ is the wheel radius.

Equation 5 refers to the ideal-optimal turning rate $\Omega$ ACKERMAN without longitudinal slipping for an Ackerman steering method. To calculate this for a skid steer vehicle $(\theta \neq 0), \theta$ must be recognized and is reflected in Equation 6. $\Omega \mathrm{W}_{\text {ACKERMAN }}$ refers to the theoretical maximum a skid steer vehicle can spin for an isotropic wheel, but, in this case, $\mathrm{F}_{\mathrm{y}}$, at $\Omega_{\text {ACKERMAN }}$, is still not zero.

$$
\sum_{\text {Ackerman }}=\frac{\omega r}{R} \cos (\theta), F y \neq 0
$$

To derive the optimal value of $\Omega_{\mathrm{Fy}=0}$, the rate the vehicle turns where these is no net $Y$ directional force, the ground velocity $V_{y}$ Figure 2B under the wheel must be equal to the velocity of the wheel ( $\omega r$ ) (no slip, which is derived in Equation 7 through 11). Equation 11 explains this relationship, and gives an upper bound of $\Omega$ for a vehicle with blank or non-directional treads. It can be easily seen from Equation 4 that, if $\mathrm{F}_{\mathrm{Y}}=0$ and $\theta \neq 0$ then $\mathrm{F}_{\mathrm{X}}$ must equal zero. Since this is impractical, for a sliding joint, $\Omega$ $\mathrm{FY}=0$ becomes an idealistic bound, with the understanding that as the lateral frictional force $\left(\mathrm{f}_{\mathrm{x}}\right)$ decreases then the spin rate $\Omega$ per $\omega$ increases

$$
V y=\cos (\theta) \text { Vground. }
$$

$$
\text { Vground }=\Omega R
$$

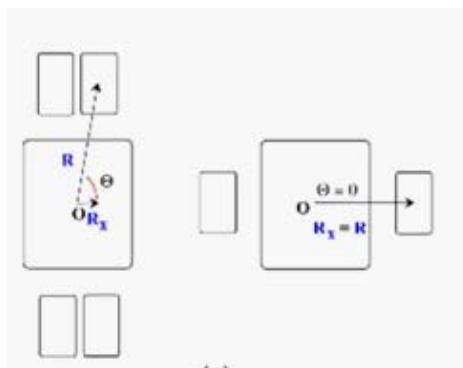

(a)

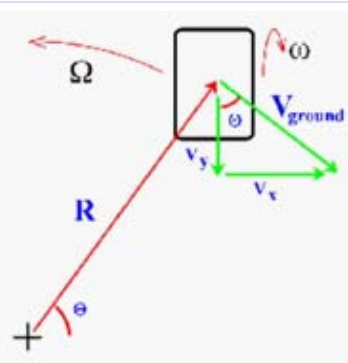

(b)
Figure 2: a) Skid Steer Geometry Configurations b) Skid Steer Kinematics.

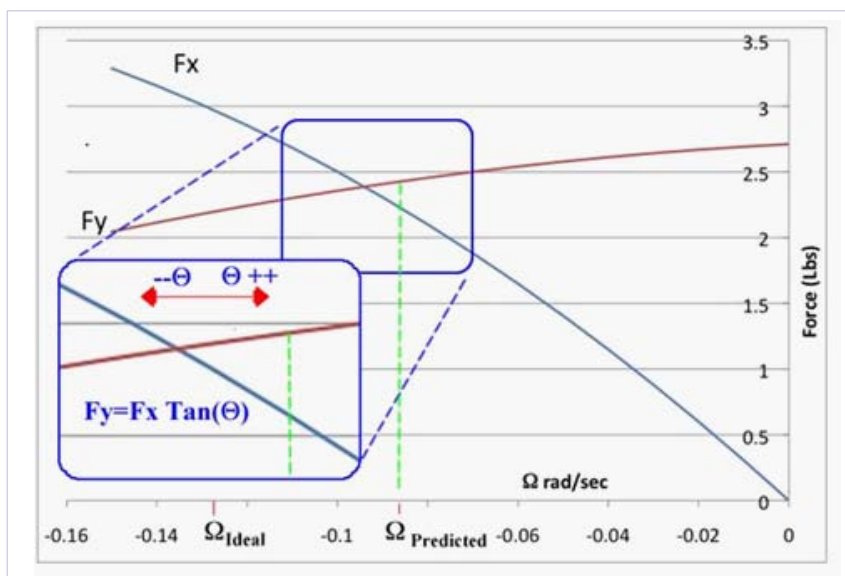

Figure 3: Force vs Spin Rate Illustration.

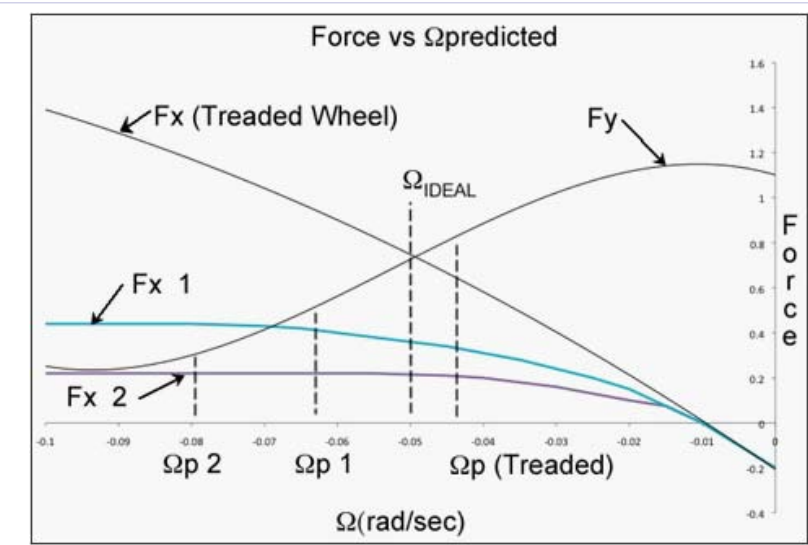

Figure 4: Fx Reduction Example. 


$$
\begin{aligned}
& \text { Vy }=\omega r \\
& \text { Vy }=\cos (\theta) \text { Vground. } \\
& \text { Vground }=\Omega R \\
& \omega r=\Omega R \cos (\theta) . \\
& \Omega F y=0=\frac{\omega r}{R \cos (\theta)}=\frac{\omega r}{R x} . \\
& \omega r=\Omega R \cos (\theta) . \\
& \Omega F y=0=\frac{\omega r}{R \cos (\theta)}=\frac{\omega r}{R x}
\end{aligned}
$$

To get a physical picture of the force behaviors between a wheel and the soil in a skid steer turn a graph can be plotted showing the forces (between the wheel and soil) as the vehicle is allowed to spin faster with a constant $\omega$ wheel spin rate. With the vehicle turning, if one was to view from the perspective of standing on the rover, the soil must pivot underneath $\left(-\Omega \frac{\mathrm{rad}}{\mathrm{sec}}\right)$. The trade off of the $\mathrm{F}_{\mathrm{x}}$ and $\mathrm{F}_{\mathrm{Y}}$ forces on the wheel can be viewed as the rate $(\Omega)$, of the ground under the wheel, increases Figure 3.

When the simulated vehicle's spin rate $(\Omega)$ is equal to zero the wheel being tested rotates ( $\mathrm{w}$ ) but does not move. This causes a force in the $\mathrm{Y}$ direction which is just the Coulomb friction $\left(\mathrm{F}_{\mathrm{y}}=\mu_{\mathrm{k}} \mathrm{N}\right)$ between the wheel and ground. (For a blank wheel on smooth ground there is no $F_{x}$ at $\Omega=0$, but for a treaded wheel $F_{x}$ could be non-zero which will be one value to focus on when testing new wheels.) As the spin rate of the ground under the wheel, increases $F_{x}$ increases. At the same time $F_{y}$ decreases until $F_{x}$ and $F_{y}$ intersect. This meeting point would represent the equilibrium spin rate ( $\Omega_{\text {Predicted }}$ ) of a square vehicle $\left(\theta=\frac{\pi}{4}\right.$ ). To find the equilibrium point, of a rectangular vehicle, Equation 4 adds the needed constraint between $\mathrm{F}_{\mathrm{x}}$ and $\mathrm{F}_{\mathrm{y}}$. For our tested vehicle (SR2 [8]) $\theta=.8477 \mathrm{rad}$ when combined with Equation 4 simplifies to Equation 12.

$$
\mathrm{F}_{\mathrm{y}=1.133 \mathrm{~F}_{\mathrm{x}}}
$$

\section{$F_{x}$ Reduction}

From the force- $\Omega$ graphs it can be seen that as $F_{x}$ is reduced $\mathrm{F}_{\mathrm{x} 1}$ and $\mathrm{F}_{\mathrm{x} 2}$ in Figure 4 along all values of $\Omega$ then the vehicle spin rate would be increased and can be explained physically as the effect of reducing the vehicle's friction in the $X$ direction. If $F_{x}$ were reduced enough then it is possible to increase the vehicle's spin rate above the Ackerman rate given by Equation 6. Also from Figure 4 it can be seen that if $\mathrm{F}_{\mathrm{Y}}$ is decreased then the vehicle spin rate will be decreased as well. So if the skid steering performance is to be enhanced then $F_{X}$ must be made independent of $F_{Y}$ and their difference broadened at the equilibrium point. It is the reduction of $F_{x}$ independently, without reducing $F_{y}$, that is an effective design goal.

\section{Validation Experiments}

To acquire the data needed to prove this concept the Suspension and Wheel Experimentation and Evaluation testbed (SWEET) was used. The testbed Figure 7A has a $3 \times 3$ meter footprint and a vertical test leg with an actuated test wheel incorporated. A six-axis, force torque sensor, is used, which stays stationary in the $\mathrm{X}$ and $\mathrm{Y}$ directions but allows movement along the Z-axis by a counterbalance design [4].

SWEET is ideal for the validation of this test since it has the ability to move in the $\mathrm{X}$ and $\mathrm{Y}$ directions, as well as pivot in the $\mathrm{X}, \mathrm{Y}$-plane. This feature gives the apparatus the unique ability to measure forces and torques in a turn allowing for the detailed data measurement of skid steer turning. In essence, what is done is operating the wheel and the ground under the wheel independently, by varying the ground speed ( $\Omega$ ), keeping the wheel spin rate $(\omega)$ constant, and observing the behavior of the forces acting on the wheel. When the forces satisfy Equation 12 the corresponding $\Omega$ is the predicted vehicle spin rate. In Figure 3 this relationship gives a point just right of the cross point and corresponds to a $\Omega$ Predicted $v$ value which is the predicted spin rate of a vehicle fitted with four wheels with the same orientation, relative to the vehicle center, and identical tread to the wheel tested. This process in an effective and time saving method for enhancing vehicle wheels in skid steering [3]. It should be noted how a vehicle's geometry affects this relationship. As $\theta$ increases

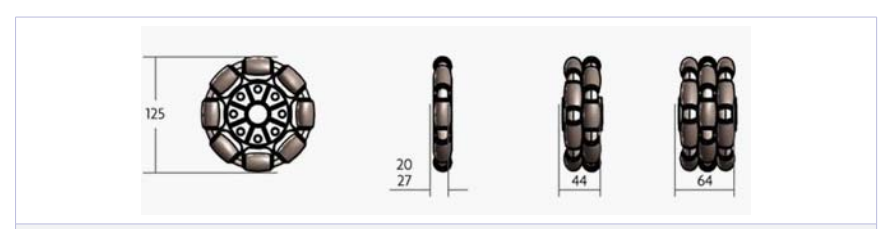

Figure 5: Omni Wheel.

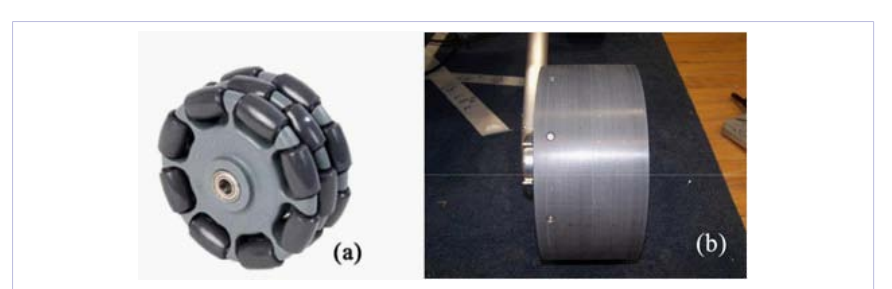

Figure 6: a) Omni-wheel [1] b) Blank Wheel.

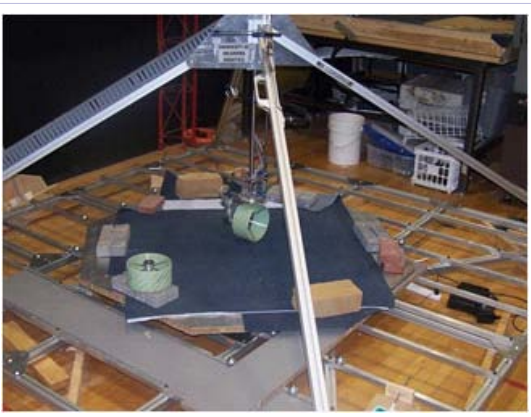

Figure 7: SWEET Testbed. 


\begin{tabular}{|c|c|c|c|c|c|c|}
\hline$\omega$ & Omni $\Omega_{\text {predicted }}$ & Blank $\Omega_{\text {predicted }}$ & Difference & Omni & Blank $\frac{\Omega}{\text { power }}$ & Difference \\
\hline $.3 \frac{\mathrm{rad}}{\mathrm{sec}}$ & -.058 & -.042 & $38 \%$. & .0034 & .0017 & $100 \%$ \\
\hline $.5 \frac{\mathrm{rad}}{\mathrm{sec}}$ & -.105 & -.064 & $64 \%$ & .005 & .002 & $150 \%$ \\
\hline
\end{tabular}

above $\frac{\pi}{4}$ the vehicle is more slender Figure 2, which makes turns less efficient, and $\Omega_{\text {predicted }}$ becomes smaller. If, on the other hand, $\theta$ decreases, its $\Omega_{\text {Predicted }}$ value increases until $\theta=0$ and $\Omega$ Predicted $=\frac{\omega r}{R}$; which is an Ackerman steering geometry.

To demonstrate that an $F_{x}$ reduction can enhance a skid steer turn, tests were run on a blank and an Omni wheel. Omni wheels Figure 5 are designed such that they do not restrict motion parallel to their axis. This is done by incorporating numerous passive wheels fixed to the outside. This added degree of freedom adds some interesting possibilities to vehicle motion such as $[9$, $2,10]$.

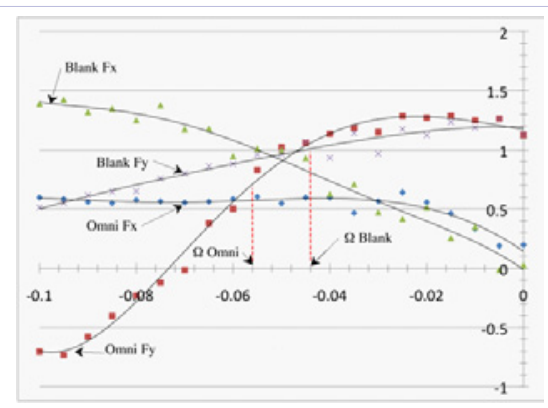

Figure 8: Results for Omni wheel spinning at $.3 \frac{\mathrm{rad}}{\mathrm{sec}}$

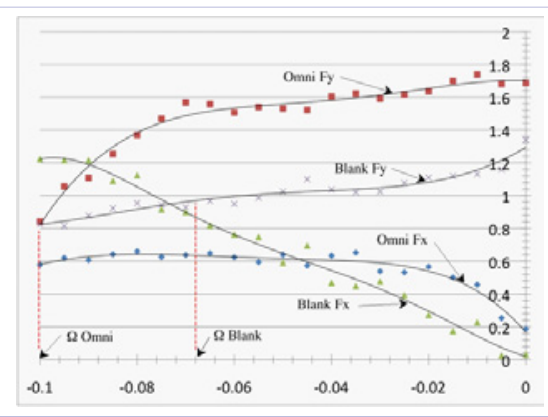

Figure 9: Results for Omni wheel spinning at .5 $\frac{\mathrm{rad}}{\mathrm{sec}}$

\section{Results}

Both the omni and blank wheels were tested at two different wheel speeds: $\mathrm{w}=0.3$ and $0.5 \frac{\mathrm{rad}}{\mathrm{sec}}$ with the range of $\mathrm{W}$ from 0 to $0.1 \frac{\mathrm{rad}}{\mathrm{sec}}$ by $.005 \frac{\mathrm{rad}}{\mathrm{sec}}$ increments. The forces that were measured were graphed in the method described above in Figure 8 that shows the $\Omega$ vehicle spin rate results for the wheel velocity of $0.3 \frac{\mathrm{rad}}{\mathrm{sec}}$ Figure 9 that gives the $\Omega$ results for $0.5 \frac{\mathrm{rad}}{\mathrm{sec}}$. These graphs illustrate the method above as well as show the difference in behavior of the two wheels. It can be seen how the $F_{X}$, the lateral force on the wheel is much flatter in higher spin rates for the omni wheel than with the blank wheel. It indicates that the frictional forces are independent of lateral motion. This behavior increases the predicted skid steer turning rate far beyond what the blank wheel predicted. The results show that the Omni wheel out performs the blank wheel in both tests even though it has a smaller diameter. In Table 1 it is shown that the omni wheel help a 38\% advantage over the blank wheel and the disparity got worse as the wheel rotational speed $\omega$ increased. To normalize the diameter difference the power usage was also measured and factored in to give turning power efficiency with units $\frac{\Omega}{\text { power }}$. This comparison further shows the difference of the two wheels' skid steering ability. For the vehicle geometry tested the Ackerman limit is 0.0433 for $\omega=0.3$ and 0.0722 for $\omega=0.5$ Equation 6 . As can be seen, the Omni wheel exceeds this value.

\section{Conclusions}

This paper discusses and demonstrates a method of increasing skid steering efficiency by independently altering the frictional components of a wheel. An Omni wheel was tested in a simulated skid steer turn and compared to a blank wheel in the same turn. As predicted the Omni wheel performed much better than the blank wheel and even turned the vehicle faster than if it had a two wheel differential drive. The data also points to the disparity between the two wheels growing larger as the wheel speed $\omega$ increases. The two tested $\omega$ of .3 and $.5 \frac{\mathrm{rad}}{\mathrm{sec}}$ showed that the Omni wheel outperformed the blank wheel by $38 \%$ and $64 \%$ respectfully.

An Omni wheel's orthogonal set minor wheels are impractical on vehicles of this type but do show that reducing $\mathrm{F}_{\mathrm{x}}$ independently 
of $\mathrm{F}_{\mathrm{Y}}$ can enhance a vehicle's skid steer turn. Swedish wheels [6] have many characteristics of Omni wheels but avoid lateral sliding of the entire vehicle. Future work will explore their effect on skid steer turn efficiency. Other factors would need to be weighed and factored depending on the vehicle's mission or directive. Such as drawbar pull, lateral sliding, and straight line efficiency.

\section{References}

1. Kornylak Corp. Omniwheel, heavy duty and lightweight multidirectional wheels for conveyors, manual and powered transfer systems, and non-powered and powered robot wheels. Technical report, 400 Heation St Hamilton, Ohio 45011.

2. Jefferey F. Four-wheel drive wheel-chair with compound wheels. Technical Report 4,823,900, United States Patent Office, 1989.

3. Daniel F, David PM, Richard H. Turning efficiency prediction for skid steer robots using single wheel testing. In FSR, 2009.
4. Daniel Flippo. Design of rover wheel testing apparatus. In ISAIRAS, 2008.

5. Daniel F, David M. Turning efficiency prediction for skid steering via single wheel testing. Journal of Terramechanics. 2014;52(1):23-29. doi: 10.1016/j.jterra.2014.02.001.

6. Bengt EI. Wheels for a course stable self-propelling vehicle movable in any desired direction on the ground or some other base. Patent 3876255, US Patent Office, 1975.

7. Matt R, David M, Zack W. Roving faster farther cheaper. In Proceedings for FSR, Chamonix, France; 2007. Field Service Robotics.

8. Matthew JR. Design and analysis of a four wheeled planetary rover. Master's thesis, University of Oklahoma. 2005.

9. Wada M. A 4wd omni directional wheelchair with a chair tilting mechanism for enhancing step climbing capability. 2008;474-479.

10. Zobova A, Tatarinov Y. Free and controlled motions of an omniwheel vehicle. Moscow University Mechanics Bulletin. 2008;63(6):146-150. 